\title{
Addressing Well-being, Burnout, and Professional Fulfillment in Child and Adolescent Psychiatry Trainees During the COVID-19 Pandemic Through Implementation of a Virtual Balint-Like Group: a Pilot Study
}

\author{
Megan D. Chochol ${ }^{1}$ (D) $\cdot$ Eric Pease $^{1} \cdot$ Cosima Swintak $^{1} \cdot$ Uma Anand $^{1}$ \\ Received: 6 November 2020 / Accepted: 13 May 2021 / Published online: 17 June 2021 \\ (C) Academic Psychiatry 2021
}

\begin{abstract}
Objective Physicians, including psychiatrists and psychiatry trainees, are at higher risk of burnout compared to the average working population. The COVID-19 pandemic heightens this risk. This pilot aims to enhance professional fulfillment and support while decreasing risk and prevalence of burnout in Child and Adolescent Psychiatry (CAP) trainees through virtual delivery of a Balint-like group incorporating brief emotional awareness modules.

Methods Six CAP trainees participated. Eight 60-min sessions held every 2 weeks were co-facilitated by a psychologist and psychiatrist who developed the curricular content. Five of the eight semi-structured sessions combined a brief emotional awareness enhancing module with a Balint-based approach to case review. The authors assessed trainee well-being, professional fulfillment, and sense of professional support pre- and post-intervention with the Well-being Index (WBI), Stanford Professional Fulfillment Index (PFI), and the authors' own supplemental survey. Descriptive statistics were reported.

Results Trainees found the curriculum feasible and useful. Surveys showed a reduction in burnout from three to zero participants $(p=0.03)$ and specific improvements in enthusiasm $(p=0.013)$, empathy with colleagues $(p=0.093)$, and connectedness with colleagues $(p=0.007)$ and patients $(p=0.042)$ at work. There were also improvements in happiness $(p=0.042)$ and valued contributions at work $(p=0.004)$.

Conclusions A novel well-being curriculum focused on combining brief emotional awareness enhancing modules with a Balintlike approach enhances professional fulfillment and a sense of professional support and decreases the risk and prevalence of burnout, even when delivered virtually to a group of CAP fellows. Results support the planned expansion of this low-cost, highvalue intervention for trainee well-being.
\end{abstract}

Keywords Virtual Balint $\cdot$ Trainee $\cdot$ Psychiatry $\cdot$ Well-being $\cdot$ Curriculum

The COVID-19 pandemic has challenged our healthcare system and the clinicians within it. Social distancing has disrupted typical onboarding and community-building activities for new and continuing trainees. Simultaneously, remote patient care and didactics have limited formal and informal opportunities for peer interaction and mentor support. Frontline heath care workers are reporting symptoms of depression, anxiety, and burnout in the setting of COVID-19 [1] and trainees may be particularly impacted [2].

Megan D. Chochol

Chochol.Megan@mayo.edu

1 Mayo Clinic, Rochester, MN, USA
Physicians and physicians-in-training already had higher rates of burnout compared to the average working population prior to the COVID-19 pandemic, and psychiatrists and psychiatry trainees face unique risk factors for burnout in their profession [3]. Physician burnout is a work-related syndrome involving emotional exhaustion, depersonalization, and a sense of reduced personal accomplishment [4]. This affects approximately $50 \%$ of both physicians-in-training and practicing physicians in all specialties, and can lead to inadequate patient care, ineffectiveness professionally, and harm to physicians, including, but not limited to, substance abuse, clinical depression, and suicidality [5]. The largest meta-analysis to date found that both individual (including mindfulness training, stress reduction approaches) and structural or organization interventions (including change in duty hour requirements) resulted in significant reductions in burnout [5]. 
Balint groups originated in primary care settings and have been widely used worldwide to provide a forum for clinicians to gather with peers to reflect on emotions aroused by challenging doctor-patient interactions [6]. Six to twelve members led by one to two group leaders comprise each group, and groups meet weekly to monthly, sometimes for years. An individual presents a case, then other group members comment on the case focusing on the emotional experience [6]. Balint groups target a variety of outcomes, including psychological mindedness, burnout and well-being, attitudes, and knowledge [6]. Balint group participation has been shown to prevent resident burnout and may help trainees become more patient-centered by improving communication skills and empathy $[7,8]$. Practicing physicians reported that participation in Balint-like groups had provided them a format to reduce isolation and process emotional interactions [9] and enhanced perceived social support among physicians working with palliative care patients [10].

Virtual facilitation and participation in Balint groups is a recent development, and to our knowledge, only one study of outcomes has been done, demonstrating significant reduction in COVID-19-related anxiety in a small group of healthcare workers in Iran [11]. There is no literature to date describing the feasibility, utility, or impact on outcomes with virtual delivery of Balint groups for trainees. We hypothesized that virtual delivery of a novel well-being curriculum focused on combining emotional awareness skills with themes from the Balint approach would enhance well-being and professional fulfillment, provide a sense of professional support, and decrease the risk and prevalence of burnout in Child and Adolescent Psychiatry (CAP) trainees.

\section{Methods}

The pilot study population included all six CAP fellows who participated in a total of eight 60 -min virtual sessions. All sessions were held after the start of the COVID-19 pandemic. In the first three sessions, facilitators addressed logistics, provided education on Balint groups and the purpose of the intervention, and obtained informed consent. The subsequent five sessions were structured in a Balint-like format. Participation was voluntary and consent to participate in the study was obtained from all six participants. Sessions were held every 2 weeks from March 2020 when the COVID-19 pandemic was initially escalating in the USA to June 2020 when the COVID-19 pandemic continued to burden healthcare workers and the general population. A clinical psychologist with the Department of Student Services and a psychiatrist trained in CAP working in the Department of Psychiatry co-facilitated all sessions. The facilitators were knowledgeable of the Balint method, however, were not formally trained in the Balint method. Neither facilitator was working with the fellows in a clinical or evaluative role. Sessions were held at noon every 2 weeks via the Health Insurance Portability and Accountability Act (HIPAA)-compliant Zoom virtual platform to promote social distancing in the setting of the pandemic.

To psychologically prime trainee participants to discuss clinical cases from an emotional perspective consistent with the Balint tradition, the group leaders developed novel 5-10min introductory modules used at the start of each session. These modules were prepared in advance and combined a stimulus activity to encourage emotional awareness with the provision of a forum for emotional expression. Content provided outlets for diverse learning styles and multiple modes of emotional expression. For each session, either a PowerPoint prompt, instant poll question, or verbal prompt was used to stimulate sharing. Some sessions encouraged participation within the larger group while others utilized breakout rooms to foster smaller group discussion. For example, when a neutral or positive stimulus was introduced such as an empty thought bubble attached to a cartoon, the entire group was involved in completing the exercise. Alternatively, when the content was more personal or provoked potentially difficult emotional experiences, breakout rooms were used. With the help of these introductory modules, participants entered a more emotionally grounded-rather than intellectually grounded-mindset.

After the emotional priming exercise, one group member volunteered to share a challenging case. No trainee was preassigned the responsibility of presenting a case; sharing was voluntary. The presenting trainee informally reviewed his or her clinical case with an emphasis on the emotional aspects of the case for approximately 5-10 $\mathrm{min}$, after which group attendees asked clarifying questions for another approximately 5-10 min. Consistent with the Balint model, the presenting trainee "exited" the group by turning off his/her camera while still observing and listening to the group discussion. For approximately $10 \mathrm{~min}$, the other trainees and the group facilitators processed the case and their emotional responses. Afterwards, the presenter returned to the group and had the opportunity to respond to the group's commentary, stimulating further discussion until the end of the hour.

The validated Well-being Index (WBI) [12] was used to assess well-being and likelihood of burnout (Table 1). The validated Stanford Professional Fulfilment Index (PFI) [13] was used to assess professional fulfillment and presence of burnout (Table 1). The authors developed supplementary survey questions to assess trainees' sense of professional support and screen for burnout (Table 2). All three of these metrics are self-report surveys containing multiple items scored on a Likert scale. The WBI derives a composite "Well-being Index score" also on a Likert scale and a composite "likelihood of burnout" score on a categorical scale comprising percent ranges (0-10\%, 11-20\%, etc.) [12]. These percent ranges 
Table 1 Validated metric results: the Well-being Index and the Stanford Professional Fulfillment Index

\begin{tabular}{|c|c|c|c|c|}
\hline & Pre-intervention mean & Post-intervention mean & Absolute change in mean & $p$-value \\
\hline \multicolumn{5}{|l|}{ Well-being Index (WBI) } \\
\hline WBI score & 4.83 & 5.33 & 0.5 & 0.296 \\
\hline Likelihood of burnout & 5.83 & 4.5 & 1.3 & 0.191 \\
\hline \multicolumn{5}{|l|}{$\begin{array}{l}\text { Stanford Professional Fulfillment Index (PFI) } \\
\text { Professional fulfillment subscale }\end{array}$} \\
\hline I feel happy at work & 1.83 & 2.67 & 0.83 & $0.042 *$ \\
\hline I feel worthwhile at work & 2.00 & 2.67 & 0.67 & 0.175 \\
\hline My work is satisfying to me & 2.83 & 3.00 & 0.17 & 0.741 \\
\hline $\begin{array}{l}\text { I feel in control when dealing with difficult } \\
\text { problems at work }\end{array}$ & 1.33 & 1.67 & 0.33 & 0.363 \\
\hline My work is meaningful to me & 3.33 & 3.50 & 0.17 & 0.695 \\
\hline $\begin{array}{l}\text { I'm contributing professionally (e.g., patient care, } \\
\text { teaching, research, and leadership) in the ways I } \\
\text { value most }\end{array}$ & 2.18 & 3.00 & 0.83 & $0.004 *$ \\
\hline \multicolumn{5}{|l|}{ Burnout subscale } \\
\hline \multicolumn{5}{|l|}{ During the past two weeks I have felt... } \\
\hline $\begin{array}{l}\text { A sense of dread when I think about work I } \\
\text { have to do }\end{array}$ & 1.50 & 1.17 & 0.333 & 0.363 \\
\hline Physically exhausted at work & 1.17 & 1.00 & 0.17 & 0.695 \\
\hline Lacking enthusiasm at work & 1.67 & 0.50 & 1.17 & $0.013 *$ \\
\hline Emotionally exhausted at work & 1.67 & 1.00 & 0.67 & 0.175 \\
\hline \multicolumn{5}{|c|}{ During the past two weeks my job has contributed to me feeling } \\
\hline Less empathetic with my patients & 0.50 & 0.33 & 0.17 & 0.695 \\
\hline Less empathetic with my colleagues & 1.33 & 0.50 & 0.83 & $0.093 *$ \\
\hline Less sensitive to others' feelings/emotions & 1.17 & 0.33 & 0.83 & $0.042 *$ \\
\hline Less interested in talking with my patients & 1.00 & 0.50 & 0.50 & 0.296 \\
\hline Less connected with my patients & 1.17 & 0.33 & 0.83 & $0.042 *$ \\
\hline Less connected with my colleagues & 1.83 & 0.33 & 1.50 & $0.007 *$ \\
\hline \multicolumn{5}{|l|}{ Dichotomous variables } \\
\hline & $\begin{array}{l}\text { No. participants } \\
\text { pre-intervention }\end{array}$ & $\begin{array}{l}\text { No. participants } \\
\text { post-intervention }\end{array}$ & $\begin{array}{l}\text { Absolute change in no. of } \\
\text { participants }\end{array}$ & $p$-value \\
\hline Burned out & 3 & 0 & 3 & $0.083 *$ \\
\hline Professionally fulfilled & 1 & 2 & 1 & 0.317 \\
\hline
\end{tabular}

*Significant to $p$-value of $<0.1$

Itemized summary of positive trends in the validated Well-being Index [13] and Stanford Professional Fulfillment Index [14]. Trends in the following items were significant: feeling happy at work, contributing professionally in the ways I value most, enthusiasm at work, empathy with colleagues, sensitivity toward others' feelings/emotions, connectedness with patients, and connectedness with colleagues. The change in the prevalence of burnout in the group from three participants to zero was significant

were converted to a corresponding 10-point Likert scale. The PFI divides items into three sections: (1) professional fulfillment, (2) work exhaustion, and (3) interpersonal disengagement. Based on responses to each Likert scale item, the PFI derives a composite score to define dichotomous burnout categories and dichotomous professional fulfillment categories [13].

These three measures were administered April 2020 prior to the first structured Balint-like session and immediately post-intervention. Data were collected anonymously using an electronic survey through the Google Forms platform. Results were analyzed using descriptive statistics and included qualitative and quantitative data. Likert scale results were averaged for each item to obtain mean scores on the pre- and post-assessment. The Google Forms platform did not track participants to pair the data; therefore, neither unpaired nor paired $t$-tests accurately compare pre- and post-intervention data. However, given data were collected from the same individuals pre- and post-intervention and the small sample size, data were assumed to be paired and paired $t$-tests were utilized to compare pre- and post-intervention item means and determine statistical significance with a $p$-value $<0.1$. The Institutional Review Board (IRB) confirmed this project was exempt from IRB review. One group facilitator held the 
Table 2 Supplementary survey results

\begin{tabular}{|c|c|c|c|c|}
\hline & Pre-intervention mean & Post-intervention mean & $\begin{array}{l}\text { Absolute change } \\
\text { in mean }\end{array}$ & $p$-value \\
\hline I feel burned out from my work & 3.33 & 2.17 & 1.17 & $0.033 *$ \\
\hline A doctor's emotions influence the doctor-patient relationship & 4.67 & 5 & 0.33 & 0.175 \\
\hline $\begin{array}{l}\text { I feel confident identifying transference and counter-transference } \\
\text { and how these impact the doctor-patient relationship }\end{array}$ & 4 & 4.5 & 0.5 & $0.076^{*}$ \\
\hline $\begin{array}{l}\text { I feel confident using my understanding of the doctor-patient } \\
\text { relationship to be effective and therapeutic with patients }\end{array}$ & 4.17 & 4.83 & 0.67 & 0.234 \\
\hline $\begin{array}{l}\text { I have access to a setting where I can process difficult cases } \\
\text { openly, without fear of judgment or retaliation }\end{array}$ & 3.67 & 4.83 & 1.17 & 0.158 \\
\hline $\begin{array}{l}\text { I am comfortable providing feedback to peers/colleagues on } \\
\text { their clinical work }\end{array}$ & 3.17 & 4.83 & 1.17 & $0.034 *$ \\
\hline The work of a psychiatrist is emotionally demanding & 4.83 & 4.83 & 0 & $\mathrm{~N} / \mathrm{A}$ \\
\hline I feel supported by my peers at work & 4 & 5 & 1 & 0.111 \\
\hline
\end{tabular}

* Significant to $p$-value of $<0.1$

Itemized summary of positive trends in the supplemental survey. Trends in the following items were significant: feeling burned out from work, confidence identifying transference and counter-transference and how these impact the doctor-patient relationship, comfort providing feedback to peers/colleagues on their clinical work

position of general psychiatry residency program director and trained four of the six participants. Otherwise, there are no conflicts of interest to report.

\section{Results}

All six child and adolescent psychiatry fellows completed preand post-implementation measures. We describe the results from each scale below.

No significant impact was seen in the overall trainee WBI scores in terms of likelihood of burnout or current well-being score. Nevertheless, there were tentative trends toward an improvement in overall WBI score and a decrease in risk of burnout (Table 1).

The validated Stanford PFI showed a statistically significant improvement in two survey items within the professional fulfillment subscale, feeling happy at work $(p=0.042)$ and feeling as though they were contributing professionally in ways which they value most $(p=0.004)$. There was no meaningful change in the other four items. With respect to the Stanford PFI burnout subscale, trainees reported significant improvement in five items: enthusiasm at work ( $p=0.012)$, feeling more empathetic towards colleagues at work ( $p=$ $0.093)$, greater sensitivity to the feelings and emotions of others $(p=0.042)$, improved feelings of connectedness with patients $(p=0.042)$, and feeling more connected to colleagues $(p=0.007)$. There was no meaningful change in the other five items. Overall, there was a decrease in prevalence of burnout in the group from three of the six subjects to none of the six subjects $(p=0.083)$. Furthermore, one additional participant met criteria for professional fulfillment post-intervention compared to pre-intervention, raising the total number of professionally fulfilled fellows to two; however, this change was not significant (Table 1).

Supplemental survey questions showed statistically significant improvement in trainees' report of feeling burned out from their work ( $p=0.033$ ), confidence in identifying how transference and counter-transference impact doctor-patient relationships $(p=0.076)$, and comfort providing feedback to peers on clinical work ( $p=0.034)$. Although not statistically significant, there was an improvement in trainee report of access to a setting where difficult cases could be processed openly, without fear of judgment or retaliation (Table 2).

Narrative feedback revealed all six fellows found the virtual Balint-like group to be a positive and beneficial experience. They also all agreed that the twice-weekly schedule was ideal. All participants advocated to continue the group through the next academic year for CAP trainees, and furthermore, advocated to expand the intervention to all psychiatry and psychology trainees within the department's nine training programs.

\section{Discussion}

Medical education has embraced expanding social media and video conferencing technologies in the setting of COVID-19 restrictions and institutional organizations are guiding program directors in these unprecedented times, as evidenced by online content dedicated to COVID-19 [14, 15]. Training programs can use these technologies in innovative ways to mitigate the isolating effects of COVID-19 on trainees. The authors implemented a Balint-like group in a new way using a 
video conferencing platform to augment social support and a sense of community at work, which are main drivers of burnout and professional engagement in physicians [16]. The novel curriculum focused on combining emotional awareness skills with themes from the Balint approach does have the potential to enhance well-being and professional fulfillment, provide a sense of professional support, and decrease burnout risk and prevalence in CAP trainees. Even though the curriculum was delivered virtually, results align with positive findings from a small Balint group initiative in psychiatry trainees that was done in person [17] and a small virtual Balint group of family doctors [18]. Positive trends from the WBI were not as strong as the improvements in burnout shown by the PFI and our own survey question assessing burnout, which was chosen because this single item has been shown to correlate with the Maslach Burnout Inventory [19]. We anticipate statistically significant trends in improved well-being and reduced burnout risk will be observed in a larger sample size.

The main limitation of the pilot is the small sample size with only six subjects, limiting statistical power. Additionally, this is a small series of eight sessions, only five of which were structured in the Balint-like format. Importantly, there is no comparison or control group to support the efficacy of the Balint-like group for the measured outcomes. Furthermore, we cannot separate the impact of the virtual novel curricular component and the impact of the virtual Balint-like component on the overall results. Although there is no literature to our knowledge comparing the efficacy of a Balint group to a placebo group (virtual or in vivo), psychotherapeutic modalities are documented to demonstrate a placebo effect of approximately $40 \%$, and a similar effect may have impacted our results [20]. Along these lines, there is also no literature to our knowledge on the impact of unstructured virtual support groups for physician burnout; however, the simple act of getting together as a group during the COVID-19 pandemic may have impacted our results.

An additional potentially confounding factor is that four of the six child and adolescent psychiatry fellows completed their general psychiatry residency at the same institution and had prior professional experience with one of the group facilitators. This prior experience may have led these trainees to feel more readily comfortable around this group facilitator compared to other trainees, positively impacting the results. Alternatively, these trainees may have felt less comfortable around this group facilitator, in turn hindering participation and negatively impacting the results. We also recognize other confounding variables may be at play, from work-related variables such as current trainee rotations to non-work-related variables such as the evolution of the COVID-19 pandemic and the transition to warmer weather in the spring/summer.

The results are correlative and conclusions cannot be drawn; however, results do support expansion of this well- being curriculum to all Psychiatry and Psychology trainees. A larger sample size will permit higher quality study design and improved statistical power to guide further expansion to trainees across disciplines. The authors received an institutional grant to provide formal training in the Balint method through the American Balint Society to ten volunteer faculty members. This group of trained faculty will maintain fidelity to the Balint tradition and serve as an ongoing resource by leading groups and by educating other faculty who wish to lead groups. Furthermore, the authors anticipate engagement in the Balint training program, which includes participation in an embedded Balint group, will enhance social connectedness and a sense of professional support among participating faculty during the COVID-19 pandemic.

Acknowledgements Center for Clinical and Translational Science Service Center, Mayo Clinic.

\section{Declarations}

The Mayo Clinic Institutional Review Board (IRB) acknowledges that based on the responses submitted for this new activity through the Mayo Clinic IRBe Human Subjects Research Wizard tool, and in accordance with the Code of Federal Regulations, 45 CFR 46.102, the above noted activity does not require IRB review.

Disclosures One author is a current general psychiatry residency program director. Otherwise, the authors have nothing to disclose.

\section{References}

1. Shreffler J, Petrey J, Huecker M. The impact of COVID-19 on healthcare worker wellness: a scoping review. West J Emerg Med. 2020;21(5):1059-66.

2. Richards M, DeBonis K. Psychiatric training during a global pandemic: how COVID-19 has affected clinical care, teaching, and trainee well-being. Psychiatr Serv. 2020;71(12):1300-2.

3. Summers RF, Gorrindo T, Hwang S, Aggarwal R, Guille C. Wellbeing, burnout, and depression among North American psychiatrists: the state of our profession. Am J Psychiatry. 2020;177(10): 955-64.

4. Maslach C, Jackson SE, Leiter MP. Maslach burnout inventory manual. Palo Alto, Calif: Consulting Psychologists Press, 1996. Print. .

5. West CP, Dyrbye LN, Erwin PJ, Shanafelt TD. Interventions to prevent and reduce physician burnout: a systematic review and meta-analysis. Lancet. 2016;388(10057):2272-81.

6. Van Roy K, Vanheule S, Inslegers R. Research on Balint groups: a literature review. Patient Educ Couns. 2015;98(6):685-94.

7. Yazdankhahfard M, Haghani F, Omid A. The Balint group and its application in medical education: a systematic review. J Educ Health Promot. 2019;8:124.

8. Huang L, Harsh J, Cui H, Wu J, Thai J, Zhang X, et al. A randomized controlled trial of Balint groups to prevent burnout among residents in China. Front Psychiatry. 2019;10:957.

9. Schwartz R, Shanafelt TD, Gimmler C, Osterberg L. Developing institutional infrastructure for physician wellness: qualitative insights from VA physicians. BMC Health Serv Res. 2020;20(1):7. 
10. Popa-Velea O, Trutescu CI, Diaconescu LV. The impact of Balint work on alexithymia, perceived stress, perceived social support and burnout among physicians working in palliative care: a longitudinal study. Int J Occup Med Environ Health. 2019;32(1):53-63.

11. Kiani Dehkordi M, Sakhi S, Gholamzad S, Azizpour M, Shahini N. Online Balint groups in healthcare workers caring for the COVID19 patients in Iran. Psychiatry Res. 2020;290:113034.

12. Dyrbye LN, Satele D, Sloan J, Shanafelt TD. Ability of the physician well-being index to identify residents in distress. J Grad Med Educ. 2014;6(1):78-84.

13. Trockel M, Bohman B, Lesure E, Hamidi MS, Welle D, Roberts L, et al. A brief instrument to assess both burnout and professional fulfillment in physicians: reliability and validity, including correlation with self-reported medical errors, in a sample of resident and practicing physicians. Acad Psychiatry. 2018;42(1):11-24.

14. Journal of Graduate Medical Education. COVID-19 and graduate medical education: a collection of JGME articles related to COVID19 and graduate medical education test. Available at https:// meridian.allenpress.com/jgme/pages/COVID-19. Last accessed: 20 January 2020.
15. Accreditation Council for Graduate Medical Education. ACGME response to pandemic crisis. Available at: https://www.acgme.org/ COVID-19. Last acccessed: 20 January 2021.

16. Shanafelt TD, Noseworthy JH. Executive leadership and physician well-being: nine organizational strategies to promote engagement and reduce burnout. Mayo Clin Proc. 2017;92(1):129-46.

17. McKensey A, Sullivan L. Balint groups - helping trainee psychiatrists make even better use of themselves. Australas Psychiatry. 2016;24(1):84-7.

18. Nease DE Jr, Lichtenstein A, Pinho-Costa L, Hoedebecke K. Balint 2.0: a virtual Balint group for doctors around the world. Int $\mathbf{J}$ Psychiatry Med. 2018;53(3):115-25.

19. West CP, Dyrbye LN, Sloan JA, Shanafelt TD. Single item measures of emotional exhaustion and depersonalization are useful for assessing burnout in medical professionals. J Gen Intern Med. 2009;24(12):1318-21.

20. Enck P, Zipfel S. Placebo effects in psychotherapy: a framework. Front Psychiatry. 2019;10:456.

Publisher's Note Springer Nature remains neutral with regard to jurisdictional claims in published maps and institutional affiliations. 